\title{
Bed rest, physiotherapy, and continuation of activities of daily living were equally effective for sciatica
}

\author{
Hofstee DJ, Gijtenbeek JM, Hoogland PH, et al. Westeinde Sciatica Trial: randomized controlled study of bed rest and \\ physiotherapy for acute sciatica.J Neurosurg (Spine) 2002 Jan;96:45-9.
}

\section{QUESTION: In patients with sciatica of $<1$ month of duration, is treatment with bed rest or physiotherapy more effective than continuation of activities of daily living (ADLs)?}

\section{Design}

Randomised (unclear allocation concealment*), unblinded,* controlled trial with 6 months of follow up.

\section{Setting}

Outpatient clinic of a university medical centre in the Hague, the Netherlands.

\section{Patients \\ 250 patients $<60$ years of age (mean age 39 y, $60 \%$ men) who were referred by general practitioners for sci- atica with radicular pain lasting $<1$ month. Exclusion criteria were the cauda equina syndrome, severe weakness, or previous bed rest or physiotherapy. 225 patients $(90 \%)$ were included in the 6 month follow up.}

\section{Intervention}

Patients were allocated to bed rest $(n=84)$, physiotherapy $(n=83)$, or continuation of ADLs $(n=83)$. Bed rest could be done at home or in the hospital and lasted 7 days. Physiotherapy sessions were scheduled twice weekly for 4 to 8 weeks and involved segmental mobilisation, disc unloading and loading exercises, and hydrotherapy. Patients in the continuation-of-ADLs group continued their jobs, household activities, and hobbies to the best of their abilities and adjusted the intensity, frequency, and duration of these activities according to the pain they felt.

\section{Main outcome measures}

Main outcomes were radicular pain (visual analogue pain scale [VAPS]) and hampered ADLs (Quebec Disability Scale [QDS]). Secondary outcomes were treatment failure (intolerable pain requiring surgery) and indications for surgery. Dr D J Hofstee, St Jansdal Hospital,

Harderwijk,

Netherlands.

DJ.Hofstee@

StJansdal.nl.

\section{Main results}

Analysis was by intention to treat. Groups did not differ for change from baseline on the VAPS or QDS at any

Bed rest, physiotherapy (Phys), or continuation of activities of daily living (ADLs) for sciatica at 6 monthst

\begin{tabular}{llllll} 
& & \multicolumn{3}{l}{$\begin{array}{l}\text { Mean improvement from } \\
\text { baseline score }\end{array}$} & Differences $(95 \% \mathrm{Cl})$ \\
\cline { 2 - 6 } Outcomes & Bed rest & Phys & ADLs & Bed rest v ADLs & Phys v ADLs \\
VAPS score & 48.2 & 46.8 & 47.8 & $0.5(-8.4$ to 9.3$)$ & $-1.0(-10.0$ to 8.0$)$ \\
\hline QDS score & 32.7 & 34.6 & 35.4 & $-2.7(-10.2$ to 4.8$)$ & $-0.7(-8.4$ to 6.9$)$ \\
\hline
\end{tabular}

†QDS $=$ Quebec Disability Scale (20 ADL items with score for each ranging from 0 [[not difficult at all]] to 5 [[unable to do]]); VAPS = visual analogue pain scale (score range 0 [no pain] to 100 [maximum pain]). All comparisons are not significant.

follow up time point (6 mo results in table). Groups did not differ for treatment failure (results at 6 mo: bed rest $v$ ADLs odds ratio [OR] $1.6,95 \%$ CI 0.8 to 3.5 ; physiotherapy $v$ ADLs OR 1.5 , CI 0.7 to 3.2) or need for surgery (results at 6 mo: bed rest $v$ ADLs OR 1.5, CI 0.7 to 3.6; physiotherapy $v$ ADLs OR 1.2, CI 0.5 to 2.9).

\section{Conclusion}

In patients with sciatica of $<1$ month of duration, bed rest or physiotherapy was not more effective than continuing activities of daily living in reducing pain, disability, and the need for surgery.

*See glossary.

\section{COMMENTARY}

Hofstee et al defined sciatica as radicular pain in $1 \mathrm{leg}$ accompanied by $\geq 1$ neurological sign. Computed tomography of the lumbosacral spine was done on all patients and showed that only $58 \%$ to $63 \%$ had radicular compression. Patients were excluded if sciatica had been present for $>1$ month. $36 \%$ of patients had a history of sciatica.

Overall pain and disability scores improved with time in all groups. The groups did not differ in these scores or in treatment failure, defined as surgery or pain so severe that patients considered surgery ( $30 \%$ to $44 \%$ ). Follow up was not ideal because 25 patients dropped out of the study.

In this study, it is possible that a longer period of bed rest (eg, until pain and the straight-leg raising sign were greatly improved) might have produced different results and conclusions. One limitation of the study was that investigators were unable to assess compliance with bed rest or use of bed rest among patients in the control group. More specific selection of patients; variation in the length and strictness of bed rest; and controlled, independently determined (by a research assistant) compliance assessments are needed before it can be concluded that bed rest is of no use for sciatica.

This study has a design similar to that of Malmivaara $e t$ $a l{ }^{1}$ which compared the effectiveness of 2 days of bed rest, back mobilising exercises, and continuation of usual activities for non-specific acute low back pain. They found that continuation of usual activities was best in promoting recovery.

Stanley L Wiener, MD

University of Illinois College of Medicine at Chicago Chicago, Illinois, USA

1 Malmivaara A, Häkkinen U, Aro T, et al. The treatment of acute low back pain: bed rest, exercises, or ordinary activity? N Engl J Med 1995;332:351-5. 